\title{
Effect of gamma irradiation on the oxygen barrier properties in ethyl-vinyl acetate/ethylene-vinyl alcohol/ ethyl-vinyl acetate multilayer film
}

\author{
Samuel Dorey ${ }^{1}$ (1) | Fanny Gaston ${ }^{1}$ ｜ Nina Girard-Perier ${ }^{1,2,3}$ ( ) | \\ Nathalie Dupuy $^{2}$ | Sylvain R.A. Marque ${ }^{3}$ ( ) | Lucie Delaunay ${ }^{1}$
}

${ }^{1}$ Sartorius Stedim FMT S.A.S, Z.I. Les Paluds, Aubagne Cedex, France

${ }^{2}$ Aix Marseille Univ, Avignon Université, CNRS, IRD, IMBE, Marseille, France

${ }^{3}$ Aix Marseille Univ, CNRS, ICR, case 551, Marseille, France

Samuel Dorey and Lucie Delaunay, Sartorius Stedim FMT S.A.SZ.I. Les Paluds, 13781 Aubagne Cedex, France. Email: samuel.dorey@sartorius.com (S.D.) and lucie.delaunay@sartorius.com (L.D.)

\begin{abstract}
The potential changes of single-use plastic materials (EVA/EVOH/EVA multilayer film in this study) used in biopharmaceutical and food-packaging industries are investigated after several gamma irradiation doses: 30, 50, 115, and $270 \mathrm{kGy}$, and for nonsterilized samples (0 kGy) from a point of view of mechanical properties, thermal properties and permeability properties. Tensile tests, differential scanning calorimetry (DSC), oxygen transmission rate (OTR) and water vapor transmission rate (WVTR) are performed on the multilayer film. For irradiation doses below $50 \mathrm{kGy}$, thermal and water vapor barrier properties are not altered. For higher doses (50 to $270 \mathrm{kGy}$ ), mechanical, thermal and water vapor barrier properties are slightly altered whereas oxygen barrier property decreases from 13 to $27 \mathrm{~cm}^{3} \cdot \mathrm{m}^{-2}$.day ${ }^{-1}$. This slight change is shown not to happen due to chain entanglement or chain mobility loss in the amorphous phase of the different polymers. It rather comes from a change of the chemical environment of the EVOH layer.
\end{abstract}

\section{KEYWOR D S}

degradation, irradiation, mechanical properties, packaging, thermal properties

\section{1 | INTRODUCTION}

Plastic packaging films are currently used in food and biopharmaceutical industries and are often multilayer structure combining oxygen and water barrier properties. The integrity and the security of these plastic films are due to appropriate flexible and barrier polymeric materials, such as polyethylene (PE) or ethylene vinyl acetate (EVA) and polyethylene-co-vinyl alcohol (EVOH). After their manufacturing, plastic films are sterilized for the biopharmaceutical industry by ionizing irradiations to reach the sterility assurance level (S.A.L) to prevent the presence of microorganisms. The current common ionizing radiation is gamma ray. The major advantage of gamma irradiation emitted from ${ }^{60} \mathrm{Co}$ source is its high penetration capacity. The treatment is usually carried out at atmospheric pressure and ambient temperature. However, gamma irradiation does not only destroy biological entities, it also generates damages to the materials. ${ }^{[1,2]}$ The gamma-radiation can also modify the polymer network by provoking crosslinking and/or chain scission. ${ }^{[1]}$ These phenomena could occur at the same time or one after the other and could generate modifications of physical and mechanical properties. One may consider the mechanical properties fall significantly when the mechanical indices change by $25 \%$. ${ }^{[2]}$

Due to industrial issues, for several years we investigate the impact of gamma irradiation as sterilization tool 
on the chemical and physical properties of multilayers films, that is, a low-density polyethylene LDPE/EVOH/ LDPE and EVA/EVOH/EVA. These layers are selected to sandwich the $\mathrm{O}_{2}$ barrier layer $\left(\mathrm{EVOH}^{[3,4]}\right)$ with two water vapor barrier layers, ${ }^{[5,6,7]} \mathrm{PE}$ and EVA, depending on industrial requirements. These investigations were carried out using several techniques: $\mathrm{EPR},{ }^{[8]}$ Raman, ${ }^{[9]}$ XPS ${ }^{[10]}$ FTIR $^{[11,12]}$ for global analysis of films pH-metry for study of the diffusion and release (spontaneous migration of the molecules from the container into the product [30 to $270 \mathrm{kGy}$ ]). In the literature, the effect of gamma irradiation on the permeability properties depends on the polymer studied. ${ }^{[5]}$ No effect was measured at low doses for polyolefins films, ${ }^{[13]}$ and significant effects were observed for EVOH film, ${ }^{[14]}$ depending on their recipe and increasingly to high doses. It seems to be recipe dependent and no hint can be given to know if it can extrapolate to other multilayer systems. Recently, for $\mathrm{PE} / \mathrm{EVOH} / \mathrm{PE}$ multilayer film, we reported an increase in its $\mathrm{O}_{2}$ barrier property upon gamma irradiation, keeping unchanged the water barrier property as well as the mechanical properties. ${ }^{[15]}$ This result is ascribed to the generation of persistent alkyl radicals in the EVOH layer. These radicals play the role of $\mathrm{O}_{2}$ scavengers. No further clue is given to extrapolate this observation to other EVOH layer thicknesses and constitutions. Therefore, we look for the generalization of these observations and turn our interest to EVA/EVOH/EVA multilayer film, which can be used in biopharmaceutical and food packaging industries prior to freeze and thaw applications. ${ }^{[16,17]}$

\section{2 | MATERIALS AND METHOD}

\subsection{EVA/EVOH/EVA multilayer film}

The EVA/EVOH/EVA multilayer film is composed of two layers of EVA and one layer of EVOH with $56 \mathrm{~mol} \%$ of $-\mathrm{VOH}$. The film is obtained by blow extrusion. The total thickness of the multilayer film is about $360 \mu \mathrm{m}$. The structure of the entire film is depicted in Figure 1. The contact layer has the largest thickness to ensure the requested chemical compatibility and the expected overall robustness. The EVOH layer thickness was selected to reach the targeted oxygen barrier property. The outer layer protects the EVOH layer from moisture. Additives (especially phenol and phosphite derivatives ${ }^{[18-20]}$ ) are included in different layers of this film for their stabilization during the manufacturing process and during their shelf life. Two or three film lots were used for investigation. Multilayers film have been provided by Sartorius Stedim FMT S.A.S and the composition of the film is a confidential trademark.

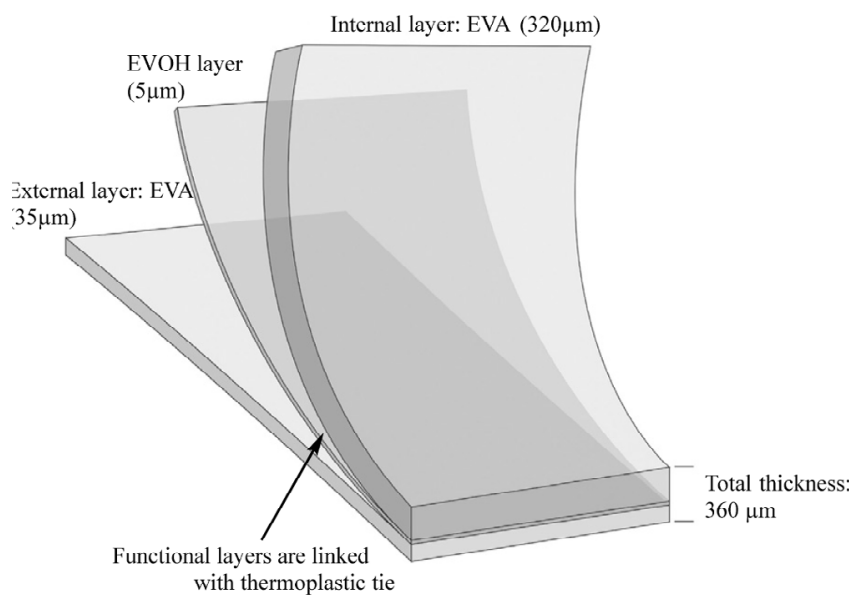

FI G U RE 1 Structure of the EVA/EVOH/EVA multilayer film. EVA, ethylene vinyl acetate; EVOH, ethylene-vinyl alcohol

\subsection{Gamma-irradiation, tensile strength testing, and gas permeability measurements}

The gamma irradiation processing, and the different measurement methodologies of the tensile strength features (UTS, elongation, toughness), the thermal properties by DSC, the OTR and the WVTR are described in a previous article. ${ }^{[15]}$ For more details see SI.

\section{3 | RESULTS}

\section{1 | Yield}

Figure 2 displays the stress-strain curve obtained either in the machine direction (MD) or in the transversal direction (TD) of the EVA/EVOH/EVA multilayer film. The stress-strain curve shows that the EVA film is a soft material according to ISO527-3. ${ }^{[15,21]}$ The mechanical properties are specified hereby by the tensile strength, the elongation at break and the toughness. Results for all lots are similar and are provided in SI.

For the non-sterile (i.e., $0 \mathrm{kGy}$ ) sample and sterile samples (i.e., 30-270 kGy) in transverse direction (Figure 2a), four zones can be defined. Two yields, at $\sim 15$ and $\sim 65 \%$ of elongation, are observed as zones I and II. From $\sim 65 \%$ elongation starts a plateau to $\sim 250 \%$ elongation for zone III. From $\sim 250 \%$ (zone IV), the strain hardening starts until the complete rupture of the film up to $\sim 600 \%$. The gamma-irradiation provokes an increase of the ultimate tensile strength at break (UTS). This increase of UTS is accompanied with a decrease of the elongation at break from $\sim 600$ down to $\sim 400 \%$. The gamma-irradiation induces a nonsignificant increase of 

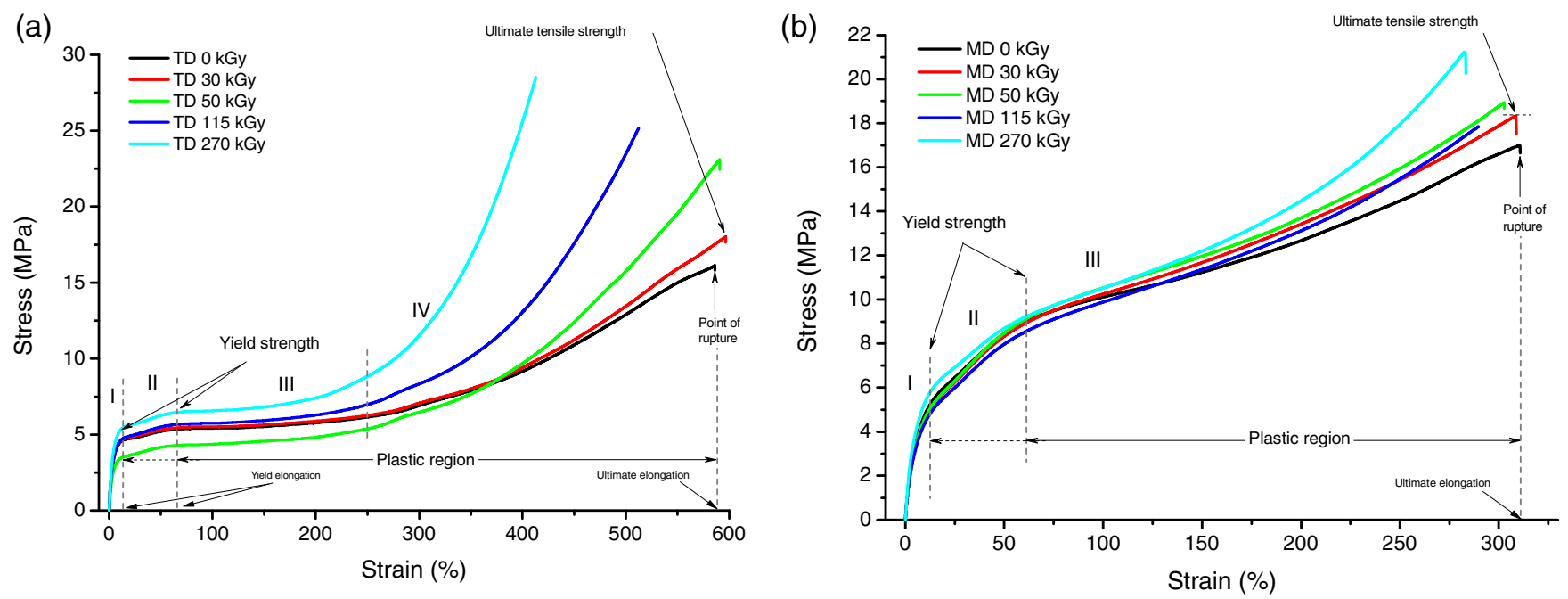

F I G U RE 2 Strain-stress curves of the EVA/EVOH/EVA multilayer film gamma-irradiated at several doses drawn in transverse direction (TD, a) and in machine direction (MD, b). One curve out of five is displayed here for each testing conditions (gamma-irradiation doses) from one lot. Other lot measurements afforded similar results and plotted in SI. EVA, ethylene vinyl acetate; EVOH, ethylene-vinyl alcohol [Color figure can be viewed at wileyonlinelibrary.com]

the first yield and a shift of the second yield. It hence seems that the whole film adapts a ductile behavior with irradiation and loose its rubber behavior especially for doses larger than $115 \mathrm{kGy}$ (Figure 2a).

For the samples in machine direction (Figure $2 b$ ), the two yields at $\sim 15$ and $\sim 60 \%$ of elongation are observed and define the phases I and II, as shown in Figure 2a. From $\sim 60 \%$ elongation, there is a continuous increase of the strength during strain (phase III). At $270 \mathrm{kGy}$, a strain hardening occurs at $\sim 200 \%$ of elongation. Increasing the gamma irradiation doses, UTS and elongation values remain the same for $0,30,50$, and $115 \mathrm{kGy}(\sim 17 \mathrm{MPa}$ for UTS and $\sim 300 \%$ for elongation) whereas the $270 \mathrm{kGy}$ induces an increase of UTS and a decrease of elongation (21 MPa for UTS and 275\% for elongation).

The difference between MD and TD figures is the absence of the phase IV in MD. The ability of a multilayer film to be stretched depends on the composition of each layer. The inner and outer layers made of EVA mainly contributes to the overall resistance of the network while the EVOH layer tends to rigidify it and to break earlier even though H-bonding are present between EVOH chains. The elastic deformation in these polymers results from the capability of the chain to stretch and to distort during the strain without covalent chain breaking. When the strain is removed, recovery from this distortion is almost instantaneous. Figure 3 shows the deformation process in a semicrystalline polymer. In contrary, the chains slide, stretch, rotate, and disentangle under load and yield the polymer to deform plastically, causing permanent deformation. As seen in Figure 2, the stress continuously increases beyond the yield point. This is because the initially tangled and intertwined chains become straight and untangled. At this point strong Van der Waals interactions between the more closely aligned chains requires higher strain in order to complete the deformation and fracture process. ${ }^{[22]}$ It would mean that the H-bonding and the Van der Waals interactions are progressively solicited during the TD stretching leading to a high elongation skill while during the MD stretching, due to sharp chain sliding, interactions suddenly disappear provoking an earlier fracture.

\section{2 | UTS and elongation}

The UTS of EVA/EVOH/EVA multilayer film lots irradiated at different gamma doses are plotted in Figure 4. Tensile tests were performed on films in Machine (MD) and Transverse (TD) directions.

After irradiation of EVA/EVOH/EVA multilayer film, a 23\% increase of the UTS in MD direction is observed with the increase of the gamma irradiation dose (Figure 4-left). An UTS plateau is reached between 30 and $115 \mathrm{kGy}$ and then increasing up to $270 \mathrm{kGy}$. The elongation in MD at break decreases with the increase of the gamma irradiation dose in machine direction. The drop is $\sim 15 \%$ between the non-irradiated sample and the sample irradiated at $270 \mathrm{kGy}$ (red circles-right). A simili-maximum can be seen between 0 and $115 \mathrm{kGy}$ before decreasing at $270 \mathrm{kGy}$.

Regarding the transverse direction (TD), an increase of $\sim 50 \%$ between the nonirradiated sample and the 

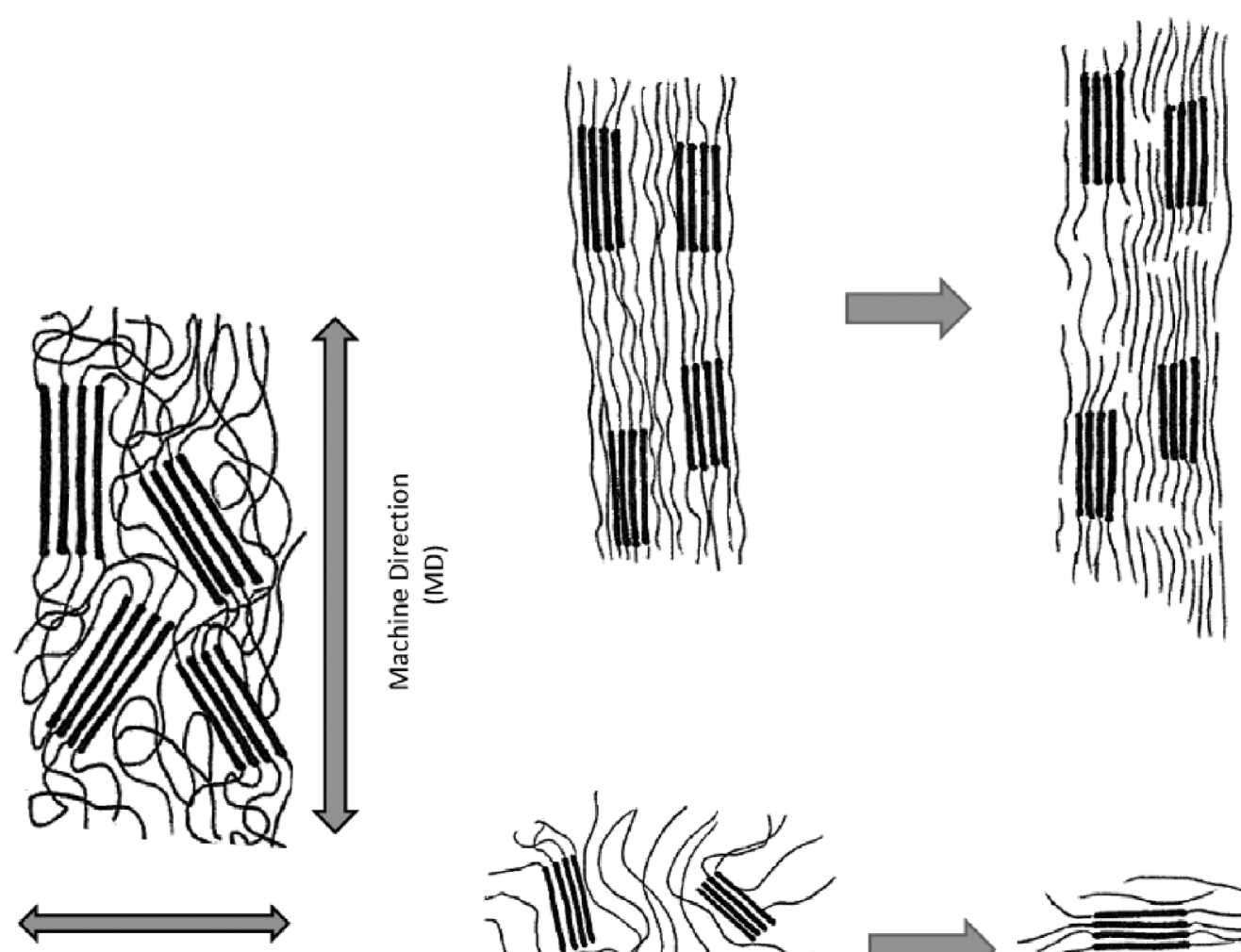

Transversal Direction

(TD)
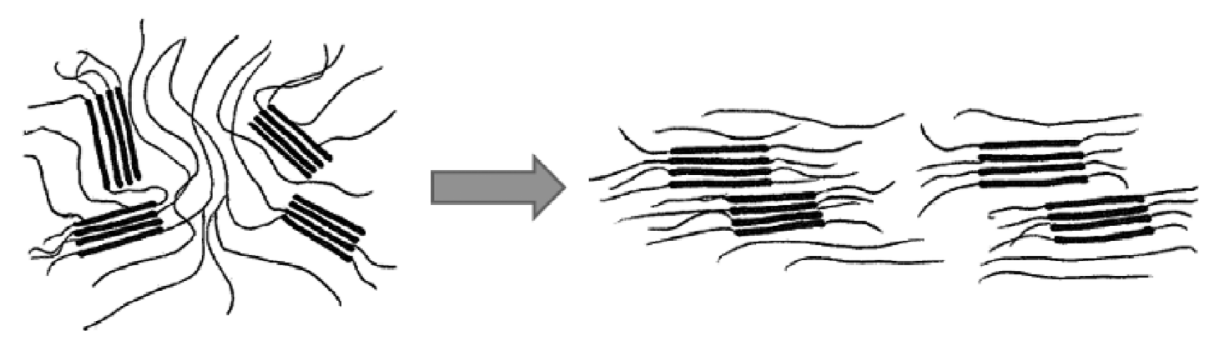

F I G U RE 3 Elongation mechanism of polymer chains in MD and TD. MD, machine direction; TD, transverse direction

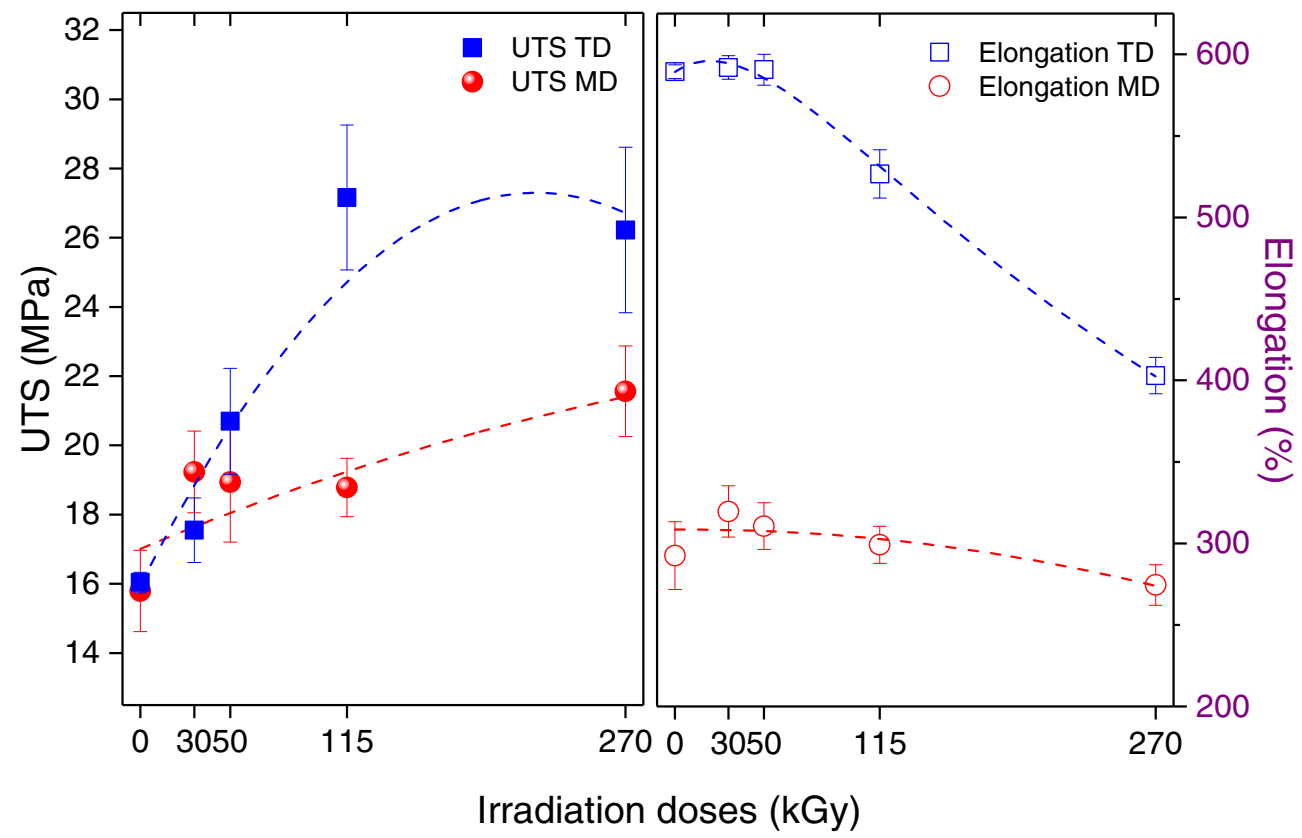

F I G U RE 4 (left) Ultimate tensile strength (UTS) for TD (blue solid squares) and MD (red solid spheres) and (right) elongation for TD (blue open squares) and MD (red open circles) as a function of the gamma-irradiation dose. Dashed fitting curves are prearranged to ease the graph reading. MD, machine direction; TD, transverse direction [Color figure can be viewed at wileyonlinelibrary.com]

sample irradiated at $115 \mathrm{kGy}$ (blue squares-left) is observed for UTS and no more influence afterward. A drop of $\sim 30 \%$ between the non-irradiated sample and the sample irradiated at $270 \mathrm{kGy}$ (blue squares-right) is observed for the elongation at break. A high lot-to-lot variation whatever the MD । TD UTS is observed, and this variation encompasses the variation of the testing method as well. 
The anisotropy of the elongation capability parallel and perpendicular to the processing direction displayed here at $0 \mathrm{kGy}$ is decreased between TD and MD at $270 \mathrm{kGy}$ compared to the nonsterile samples. The anisotropy of UTS is generated and amplified by the gamma irradiation. This tensile strength anisotropy is directly linked to the extrusion process. In the film blowing process, the filtered melt is pressed through an annular die yielding in a tubular film. ${ }^{[23]}$ Depending on the inflate of the bubble (i.e., on the film thickness) and on the cooling of the bubble, which is the time allowed for the material to crystallize, the polymer chains can be more or less oriented. However, it can be seen in Figure 5 there is no apparent spherulites and thereof no apparent preferred orientation caused by the manufacturing process indicating that the structure reorganization does not take place at the spherulite level.

In comparison with $\mathrm{PE} / \mathrm{EVOH} / \mathrm{PE}$ multilayer film in a previous study, UTS and elongation showed a slight decrease in properties at low doses (30-50 kGy) for TD mode and a slight increase in MD mode. For doses up to $270 \mathrm{kGy}$ a detrimental effect has been observed. Either EVA/EVOH/EVA or PE/EVOH/PE multilayer films become less flexible when they are gamma irradiated. ${ }^{[15]}$

\section{3 | Toughness}

The toughness values are obtained from five EVA/EVOH/EVA multilayer film lots irradiated at different gamma doses. Toughness of the film defined as the capacity to resist to a pressure, a shock and/or the wear and corresponds to the energy the film needs to break.
The toughness (or tensile energy at break, TEB) is the combination of the UTS and the elongation, and is defined by the area under the stress-strain curve in Figure 2 and calculated per Equation (1).

$$
T E B=\int_{0}^{\epsilon_{T}} S d \epsilon .
$$

Where $S$ is the stress at any strain, $\epsilon$, and $\epsilon_{T}$ is the strain at rupture. ${ }^{[24]}$

Toughness is linked to the product of elongation by UTS. The behaviors of the toughness per MD and per TD follows directly the slight modification of the UTS and elongation due to the gamma irradiation. The loss of the toughness per TD $\geq 115 \mathrm{kGy}$ is directly linked as well to the decrease of UTS and elongation while the toughness per TD does not change in the range $0-115 \mathrm{kGy}$ (Figure 6). In the range $0-270 \mathrm{kGy}$ for the MD, no obvious toughness change is observed as all differences are within recorded SD (Figure 6). The gamma irradiation in the 30-270 kGy range does not influence the robustness of the EVA film compared to the nonsterile film (i.e., $0 \mathrm{kGy}$ film). Regarding previous results obtained with another multilayer film $\mathrm{PE} / \mathrm{EVOH} / \mathrm{PE},{ }^{[15]}$ toughness was higher for both modes at non irradiated dose whereas they decreased until $40 \mathrm{~mJ} . \mathrm{mm}^{-3}$ at $270 \mathrm{kGy}$.

\section{4 | DSC}

With results from DSC, it appears, in one hand, that the $\mathrm{EVOH}$ layer melting temperature drops from $165^{\circ} \mathrm{C}$ at
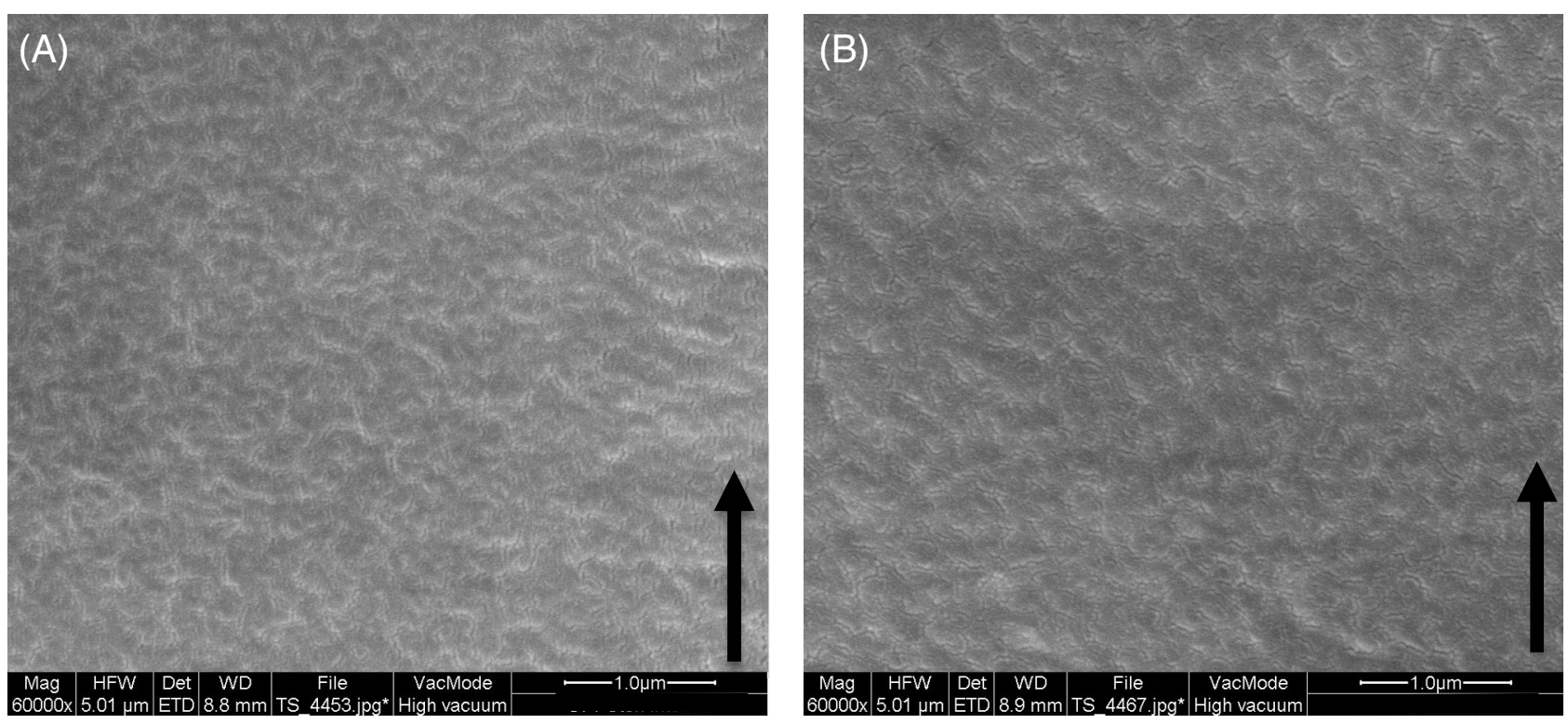

F I G U RE 5 SEM picture of the sterile (50 kGy) (a) and the nonsterile (0 kGy) (b) EVA/EVOH/EVA film (internal layer as per Figure 1). The machine direction (MD) is given by the arrow. EVA, ethylene vinyl acetate; EVOH, ethylene-vinyl alcohol 
$0 \mathrm{kGy}$ down to $150^{\circ} \mathrm{C}$ at $270 \mathrm{kGy}$ (Figure 7a). A previous study revealed that indeed the EVOH layer is the layer the most impacted by the gamma-irradiation. ${ }^{[8]}$ On the other hand, the absence of changes in melting points ( $4 \%$ drop from $0 \mathrm{kGy}$ to the $270 \mathrm{kGy}$; Figure $7 \mathrm{~b}$ ) denotes the absence of significant modifications due to gamma irradiation of EVA layers.

\section{5 | OTR-WVTR}

Interestingly, the WVTR barrier is not significantly modified by gamma irradiation, that is, $15 \%$ of increasing

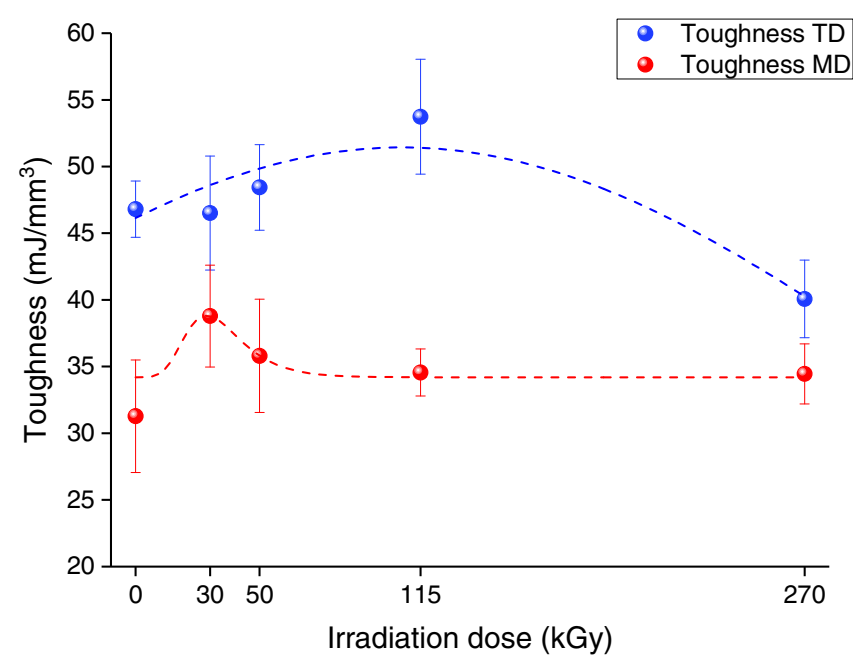

F I G U RE 6 Toughness per TD (blue spheres) and MD (red spheres) against gamma-irradiation dose. Dashed fitting curves are given to ease the reading. MD, machine direction; TD, transverse direction [Color figure can be viewed at wileyonlinelibrary.com] permeation from 0 to $270 \mathrm{kGy}$ (Figure 8a). On the other hand, and in sharp contrast with the results previously reported concerning the increasing $\mathrm{O}_{2}$ barrier property of the EVOH layer in PE/EVOH/PE multilayer film, a striking twofold increase in permeation is observed from 0 to $270 \mathrm{kGy}$ (Figure 8b). However, at doses required for industrial applications in packaging, this $\mathrm{O}_{2}$ barrier is not significantly altered.

\section{4 | DISCUSSION}

\section{1 | Mechanical properties}

The increase of UTS and the diminution of elongation when the gamma irradiation dose increases (for MD and TD; more pronounced in TD) is caused by the resistance of the multilayer film. This resistance is due to the cross linking of the polymers chains in EVA layer. ${ }^{[25]}$ Hence, EVA chains have lower mobility and the multilayer film become less flexible. Moreover, the slight decrease in melting points with gamma irradiation doses for EVA layer is in nice agreement with mechanical properties.

The $15^{\circ} \mathrm{C}$ drop of melting temperature observed for the EVOH layer (from 0 to $270 \mathrm{kGy}$ ) denotes bond breaking events either in the H-bonding network or in the backbone affording chains of lower masses and hence a greater mobility of chains, that is, less stress resistant materials. ${ }^{[26]}$ As mechanical properties are not strikingly altered, these events are not predominant contrary to the cross linking of EVA chains which participates to the mechanical resistance of the EVA/EVOH/EVA multilayer film to various strains. (a)

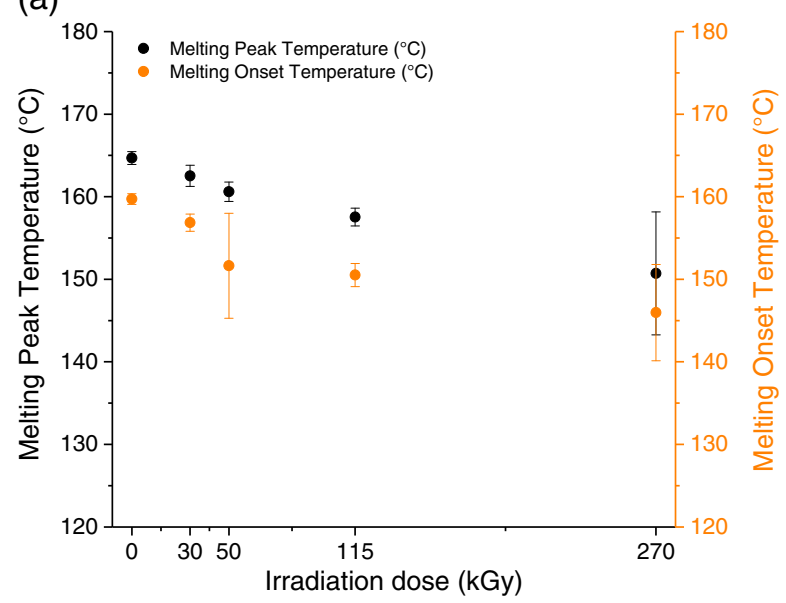

(b)

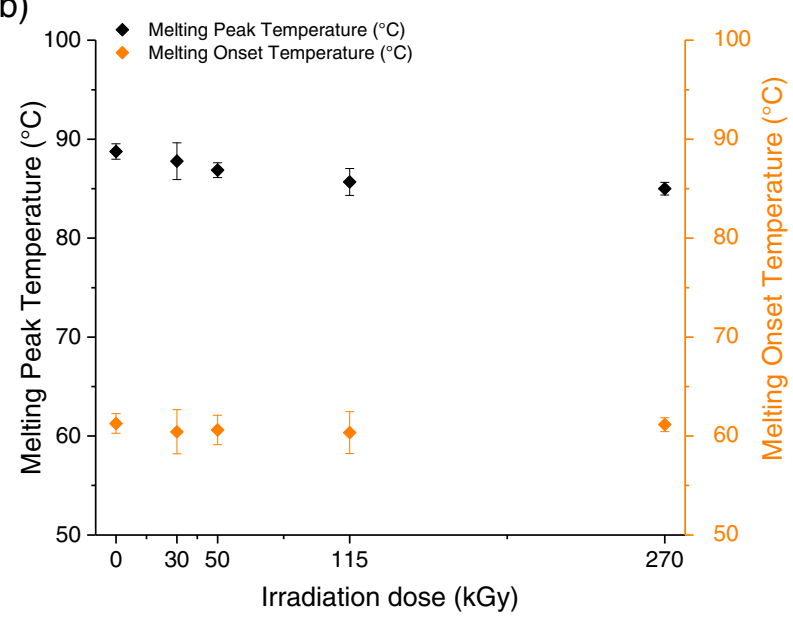

FIG URE 7 melting peaks and melting onset points for the EVOH layer (a) and EVA layer (b) used in the EVA/EVOH/EVA multilayer film according to the gamma irradiation dose. EVA, ethylene vinyl acetate; EVOH, ethylene-vinyl alcohol [Color figure can be viewed at wileyonlinelibrary.com] 
(a)

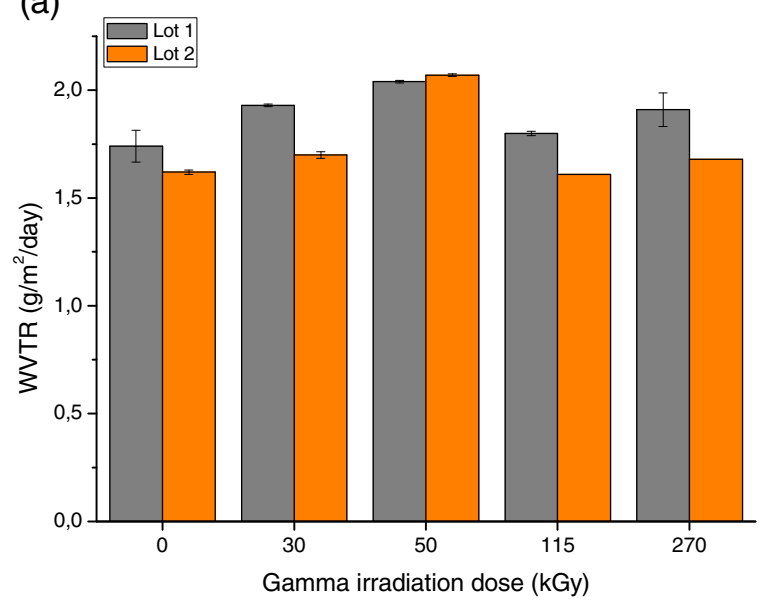

(b)

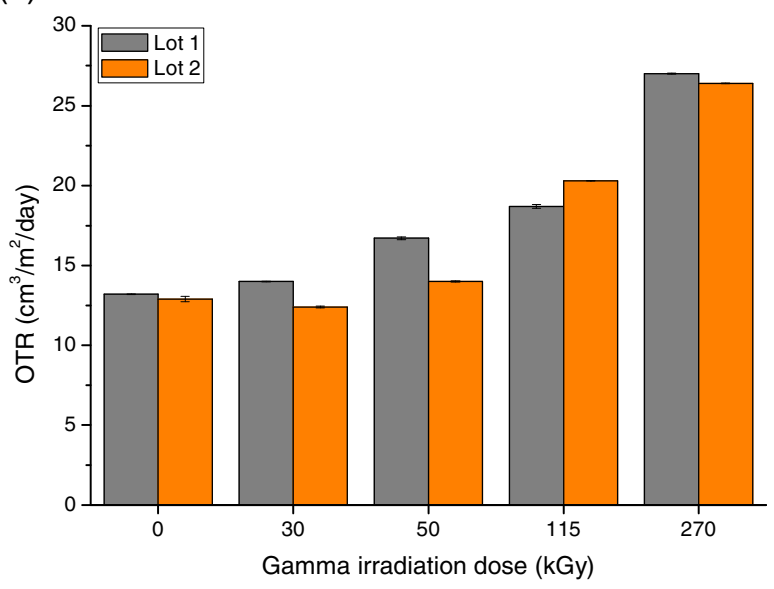

F I G U R E 8 WVTR (a) and OTR (b) of EVA/EVOH/EVA multilayer film according to the gamma irradiation dose. OTR, oxygen transmission rate; WVTR, water vapor transmission rate [Color figure can be viewed at wileyonlinelibrary.com]

\section{2 | Barriers}

$\mathrm{O}_{2}$ barrier properties of EVOH layer are among the best for materials. ${ }^{[3,4]}$ This barrier property depends on ethylene content, crystallinity, thickness, and free volume. ${ }^{[4]}$ If one of these properties is altered, the barrier effect can be detrimentally affected. The drop of $15 \%$ in melting point of EVOH layer by DSC denotes an alteration of the materials in nice agreement with the increase in $\mathrm{O}_{2}$ permeation of the materials. Indeed, gamma irradiation is expected to cleave both covalent bonds in the EVOH backbone and $\mathrm{H}$-bonding in $\mathrm{H}$-bonding network generating micro voids ${ }^{[27]}$ or micro-channels in the EVOH layer favoring the migration of $\mathrm{O}_{2}$ though the materials, and, hence, a lower $\mathrm{O}_{2}$-barrier property as displayed in Figure $8 \mathrm{~b}$. This observation is in sharp contrast with the striking enhancement in $\mathrm{O}_{2}$-barrier of $\mathrm{EVOH}$ layer reported for $\mathrm{PE} / \mathrm{EVOH} / \mathrm{PE}$ multilayer film. ${ }^{[15]}$ As $\mathrm{O}_{2^{-}}$ barrier property is due to EVOH layer and same components in both EVA/EVOH/EVA and PE/EVOH/PE multilayer films, the same degradation events are expected for the EVOH layer. Moreover, regarding the EVA/EVOH/EVA multilayer film, no radicals were detected by EPR. ${ }^{[28]}$ Hence, the striking difference in $\mathrm{O}_{2}$ barrier property between EVA/EVOH/EVA and $\mathrm{PE} / \mathrm{EVOH} / \mathrm{PE}$ multilayer films is ascribed to the difference in VOH contents (i.e., 56 vs. $71 \mathrm{~mol} \%$ ) and to the thickness (i.e., 5 vs. $25 \mu \mathrm{m}$ ) of the EVOH layer.

Indeed, this lower content in $\mathrm{VOH}$ and thinner layer in EVOH likely weakens the resistance of materials to gamma irradiation as H-bonding network is less developed and likely less strong. In addition, it lowers the amount of radicals generated in such way that radicals are not detected by EPR. As expected from mechanical and thermal properties of EVA layers, the water vapor barrier properties of EVA layers are not altered by gamma irradiation whatever the dose up to $270 \mathrm{kGy}$.

\section{5 | CONCLUSION}

These results regarding the EVA/EVOH/EVA multilayer film show that some properties depend dramatically on the doses received and on the physical material feature of interest. Importantly, in the recommended sterilization dose range (30-50 kGy) for biopharmaceutical industry, mechanical, thermal, and water vapor barrier properties are not significantly changed compared to the nonsterilized materials. It is of course true for lower doses applied to the food industry $(<15 \mathrm{kGy})$. The $\mathrm{O}_{2}$ barrier property is decreased for EVA/EVOH/EVA multilayer film whereas it is strikingly increased for another packaging film with a PE/EVOH/PE multilayer structure. ${ }^{[15]}$ This denotes a high sensitivity of this property both to composition of the EVOH polymer (VOH content) and to the structure of the film (thickness of EVOH layer). For higher doses (from 50 to $270 \mathrm{kGy}$ ) mechanical, thermal and water vapor barrier properties are slightly altered.

\section{ACKNOWLEDGMENTS}

The authors acknowledge Sartorius Stedim FMT S.A.S. for the financial support of this work.

\section{ORCID}

Samuel Dorey (1) https://orcid.org/0000-0003-3178-2212 Nina Girard-Perier (1) https://orcid.org/0000-0002-5925668X Sylvain R.A. Marque (1) https://orcid.org/0000-0002-30508468 


\section{REFERENCES}

[1] A. Chapiro, Radiat. Res. Suppl. 1964, 4, 179.

[2] V. S. Ivanov, Radiation Chemistry of Polymers, VSP, Utrecht 1992.

[3] K. K. Mokwena, J. Tang, Crit. Rev. Food Sci. Nutr. 2012, 52,640 .

[4] C. Maes, W. Luyten, G. Herremans, R. Peeters, R. Carleer, M. Buntinx, Polym. Rev. 2017, 58, 209.

[5] J. Lange, Y. Wyser, Packag. Technol. Sci. 2003, 16, 149.

[6] M. Borhani Zarandi, H. Amrollahi Bioki, Z.-A. Mirbagheri, F. Tabbakh, G. Mirjalili, Appl. Radiat. Isot. 2012, 70, 1.

[7] A. E. Goulas, K. A. Riganakos, A. Badeka, M. G. Kontominas, Food Addit. Contam. 2002, 19, 1190.

[8] G. Audran, S. Dorey, N. Dupuy, F. Gaston, S. R. A. Marque, Polym. Degrad. Stab. 2015, 122, 169.

[9] F. Gaston, N. Dupuy, S. R. A. Marque, S. Dorey, Vib. Spectrosc. 2018, 96, 52.

[10] S. Dorey, F. Gaston, S. R. A. Marque, B. Bortolotti, N. Dupuy, Appl. Surf. Sci. 2018, 427, 966.

[11] F. Gaston, N. Dupuy, S. R. A. Marque, M. Barbaroux, S. Dorey, Radiat. Phys. Chem. 2016, 125, 115.

[12] F. Gaston, N. Dupuy, S. R. A. Marque, M. Barbaroux, S. Dorey, Polym. Degrad. Stab. 2016, 129, 19.

[13] J. George, R. Kumar, V. A. Sajeevkumar, S. N. Sabapathy, S. G. Vaijapurkar, D. Kumar, A. Kchawahha, A. S. Bawa, Radiat. Phys. Chem. 2007, 76, 1205.

[14] Y. Jae Byun, S. I. Hong, K. B. Kim, D. H. Jeon, J. M. Kim, W. S. Whiteside, H. Jin Park, Radiat. Phys. Chem. 2007, 76, 974.

[15] S. Dorey, F. Gaston, N. Girard-Perier, N. Dupuy, S. R. A. Marque, L. Delaunay, Ind. Eng. Chem. Res. 2019, 58, 14115.

[16] U. T. Lashmar, M. Vanderburgh, S. J. Little, BioProc. Int. 2007, $5,44$.

[17] M. Puri, S. Morar-Mitrica, G. Crotts, D. Nesta, BioProc. Int. 2015, 13, 34.

[18] D. H. Jeon, G. Y. Park, I. S. Kwak, K. H. Lee, H. J. Park, Food Sci. Technol. 2007, 40, 151.
[19] F. Bourges, G. Bureau, J. Dumonceau, B. Pascat, Packag. Technol. Sci. 1992, 5, 205.

[20] J. Pospíšil, Polym. Degrad. Stab. 1993, 40, 217.

[21] ISO527. Plastics - Determination of tensile properties - Part 3: Test conditions for films and sheets, 1995.

[22] D. R. Askeland, The Science and Engineering of Materials, Springer, Boston, MA 1996.

[23] Ullmann's Polymers and Plastics: Products and Processes, Wiley-VCH, Weinheim 2016.

[24] ASTM D882-02. Standard Test Method for Tensile Properties of Thin Plastic Sheeting.

[25] Y. H. Gad, M. M. Magida, H. H. J. El-Nahas, Ind. Eng. Chem. 2010, 16, 1019.

[26] L. Zaidi, S. Bruzaud, M. Kaci, A. Bourmaud, N. Gautier, Y. Grohens, Polym. Degrad. Stab. 2013, 98, 348.

[27] G. Dlubek, T. Lüpke, J. Stejny, M. A. Alam, M. Arnold, Macromolecules 2000, 33, 990.

[28] G. Audran, S. Dorey, N. Dupuy, F. Gaston, S. R. A. Marque, unpublished. 